\title{
Transient Synaptic Silencing of Developing Striate Cortex Has Persistent Effects on Visual Function and Plasticity
}

\author{
Matteo Caleo, ${ }^{1 \star}$ Laura Restani, ${ }^{2 \star}$ Laura Gianfranceschi, ${ }^{2 \star}$ Laura Costantin, ${ }^{2}$ Chiara Rossi, ${ }^{1}$ Ornella Rossetto, ${ }^{3}$ \\ Cesare Montecucco, ${ }^{3}$ and Lamberto Maffei ${ }^{1,2}$ \\ ${ }^{1}$ Istituto di Neuroscienze, Consiglio Nazionale delle Ricerche and ${ }^{2}$ Scuola Normale Superiore, 56100 Pisa, Italy, and ${ }^{3}$ Dipartimento di Scienze Biomediche, \\ Università di Padova, 35121 Padova, Italy
}

\begin{abstract}
Neural circuits in the cerebral cortex are shaped by experience during "critical periods" early in life. For example, visual cortex is immature at the time of eye opening and gradually develops its functional properties during a sensitive period. Very few reports have addressed the role of intrinsic neural activity in cortical maturation. Here we have exploited the bacterial enzyme botulinum neurotoxin $\mathrm{E}$ (BoNT/E) to produce a unilateral, reversible blockade of neural activity in rat visual cortex during the sensitive period. BoNT/E is a highly selective protease that interferes with transmitter release via cleavage of the synaptic protein SNAP-25 (synaptosomal-associated protein of $25 \mathrm{kDa}$ ). Unilateral, intracortical injections of BoNT/E were made at the time of eye opening and resulted in the silencing of the treated, but not contralateral, hemisphere for a period of 2 weeks. We found that visual acuity was permanently reduced in the blocked hemisphere, and the critical period for ocular dominance plasticity persisted into adulthood. Unexpectedly, these effects extended equally to the contralateral, uninjected side, demonstrating a fundamental role for interhemispheric connections in cortical maturation.
\end{abstract}

Key words: botulinum neurotoxin; synaptic transmission; critical period; visual acuity; monocular deprivation; corpus callosum

\section{Introduction}

The mammalian visual cortex is immature at the time of eye opening and gradually develops its functional and structural properties during a critical period early in life (Sherman and Spear, 1982; Berardi et al., 2000; Sur and Leamey, 2001; Fox and Wong, 2005; Hensch, 2005). During this period of heightened plasticity, the refinement and fine-tuning of neural circuits requires visual experience (Katz and Shatz, 1996; Hensch, 2005). A number of studies have addressed the role of visual input in sculpting cortical circuitry. Monocular deprivation (MD) and dark rearing are two classic paradigms for the study of experience-dependent changes in neural connectivity. For example, animals reared in complete darkness from birth show alterations in glutamatergic and GABAergic transmission (Benevento et al., 1992; Desai et al., 2002; Morales et al., 2002) as well as a change in the threshold for synaptic potentiation and depression (Kirkwood et al., 1996). Cortical cells have large receptive fields, high rates of spontaneous discharge, and poor direction selectivity (Gianfranceschi et al., 2003; Li et al., 2006). Visual acuity remains also abnormally low (Fagiolini et al., 1994; Gianfranceschi et al., 2003).

Although the impact of afferent visual input on cortical mat-

Received Dec. 5, 2006; accepted March 13, 2007.

This work was supported by funds from the Italian Ministry for Research (Fondo per gli Investimenti della Ricerca di Base and Progetti di Rilevante Interesse Nazionale) and by Telethon Foundation Grant GGP04086 (M.C.). We are grateful to C. Orsini and G. C. Cappagli for excellent technical assistance.

${ }^{*} M$.C, L.R., and L.G. contributed equally to this work.

Correspondence should be addressed to Dr. Matteo Caleo, Istituto di Neuroscienze, Consiglio Nazionale delle Ricerche, via G. Moruzzi 1, 56100 Pisa, Italy. E-mail: caleo@in.cnr.it.

D01:10.1523/JNEUROSCI.0772-07.2007

Copyright $\odot 2007$ Society for Neuroscience $\quad$ 0270-6474/07/274530-11\$15.00/0 uration has been thoroughly investigated, only a few reports have examined the effects of blocking cortical activity during the sensitive period. Intracortical infusion of tetrodotoxin (TTX) completely prevents the maturation of orientation selectivity in ferret visual cortex (Chapman and Stryker, 1993). At the anatomical level, silencing action potentials with TTX blocks the formation of patchy horizontal connections linking columns with similar preferred orientation (Ruthazer and Stryker, 1996). These data point to a crucial role for cortical discharges in the maturation of orientation selectivity. However, the role of cortical activity in other developmental processes remains unexplored.

Here we have exploited the clostridial enzyme botulinum neurotoxin $\mathrm{E}$ (BoNT/E) to produce a long-term silencing of activity in the developing rat primary visual cortex. BoNT/E is a metalloprotease that enters the cytosol of nerve terminals close to the site of delivery and specifically cleaves the synaptic protein SNAP-25 (synaptosomal-associated protein of 25 $\mathrm{kDa}$ ), causing a prolonged blockade of transmitter release (Schiavo et al., 2000; Davletov et al., 2005). We demonstrated recently that a single injection of BoNT/E into the rat hippocampus blocks both spontaneous and evoked neural activity for at least 2 weeks (Costantin et al., 2005). Here, a unilateral injection of BoNT/E was performed into rat primary visual cortex to silence neural discharges during the sensitive period. We examined the effects of this activity blockade on development of visual function and termination of the critical period. Moreover, we assessed maturation of striate cortex contralateral to the blocked side (which maintains its direct visual input) to probe a role for interhemispheric communication in visual cortical development. 


\section{Materials and Methods}

\section{Animal treatment}

Long-Evans hooded rats were used in this study. Animals were reared in a $12 \mathrm{~h} \mathrm{light/dark} \mathrm{cycle,} \mathrm{with} \mathrm{food} \mathrm{and} \mathrm{water} \mathrm{available} \mathrm{ad} \mathrm{libitum.} \mathrm{All}$ experimental procedures conformed to the European Communities Council Directive number 86/609/EEC.

BoNT/E was obtained by Wako (Osaka, Japan), trypsin activated, purified, and tested as described previously (Schiavo and Montecucco, 1995; Costantin et al., 2005). Unilateral stereotaxic injections of BoNT/E (30 nM) or vehicle (2\% rat serum albumin in PBS) were made into left primary visual cortex of postnatal day 14 (P14) rat pups under avertin anesthesia (tri-bromo-ethanol; Sigma, St. Louis, MO). Injections were performed at three locations by means of a glass pipette $(40 \mu \mathrm{m}$ tip diameter) mounted on a motorized three-axis micromanipulator connected to an injector. The three locations were as follows: $2.5 \mathrm{~mm}$ lateral to the midline and $1 \mathrm{~mm}$ anterior to lambda; $2.5 \mathrm{~mm}$ lateral and in correspondence with lambda; $2.5 \mathrm{~mm}$ lateral and $1 \mathrm{~mm}$ posterior to lambda. At each site, $0.5 \mu$ l were slowly delivered at a depth of $1 \mathrm{~mm}$ below dura.

Monocular deprivation was performed by eyelid suture under isoflurane anesthesia. MD animals were carefully inspected every day to make sure that the lid suture remained intact.

\section{Histology}

P14 rat pups received unilateral injections of BoNT/E $(n=9)$ or vehicle $(n=5)$. One to $4 \mathrm{~d}$ later, they were deeply anesthetized and perfused through the heart with $4 \%$ paraformaldehyde. Coronal sections ( $40 \mu \mathrm{m}$ thick, cut on a freezing microtome) were reacted with antibodies recognizing either BoNT/E-cleaved or intact SNAP-25. Cleaved SNAP-25 was detected using a peptide affinity-purified polyclonal antibody raised against the BoNT/E truncated C-terminal peptide of SNAP-25 (CDMGNEIDTQNRQIDR). This antibody specifically recognizes cleaved SNAP-25 but not the whole protein (Costantin et al., 2005). Intact SNAP-25 was revealed with a polyclonal antibody raised against a synthetic peptide corresponding to residues 195-206 of SNAP-25 protein.

For immunostaining, sections were blocked with $10 \%$ normal goat serum in PBS containing $0.3 \%$ Triton X-100 and then incubated overnight at $4^{\circ} \mathrm{C}$ with the primary antibodies. For detection of cleaved SNAP25 , sections were reacted with a biotinylated secondary antibody (Vector Laboratories, Burlingame, CA), followed by avidin-biotin-peroxidase complex (ABC kit; Vector Laboratories) and diaminobenzidine reaction. For detection of intact SNAP-25, sections were incubated with AlexaFluor568-conjugated secondary antibody (Invitrogen, Carlsbad, CA).

A group of normal rats $(n=4)$ and rats unilaterally injected at P14 with vehicle $(n=4)$ or BoNT/E $(n=5)$ were perfused at P35 for the anatomical analysis. Serial cortical sections (one of six) were Nissl stained for the evaluation of cortical thickness. Additional serial sections were incubated overnight at $4^{\circ} \mathrm{C}$ with monoclonal antibodies specific for the neuronal-specific nuclear protein NeuN (1:500; Chemicon, Temecula, $\mathrm{CA})$ or the GABA biosynthetic enzyme GAD65 (1:500; Chemicon) or in a solution of biotin-conjugated wisteria floribunda agglutinin (WFA) (10 $\mu \mathrm{g} / \mathrm{ml}$ ). Bound primary antibodies were detected with biotinylated antimouse (1:200), followed by cyanine 3 (Cy3)-conjugated ExtrAvidin (1: 500; Sigma). WFA was revealed with a $1 \mathrm{~h}$ incubation in Cy3-conjugated ExtrAvidin (1:500; Sigma).

Additional rats (vehicle, $n=4$; BoNT/E, $n=4$ ) were injected unilaterally at P14 and perfused when they were older than P100. Serial cortical sections were stained with anti-GAD65 antibodies as described above.

\section{Morphometry and confocal analysis}

All data were collected blind to the experimental treatment.

Cortical thickness. The analysis was performed on coronal sections of primary visual cortex using Stereo Investigator software (MicroBrightField, Colchester, VT) and a Zeiss (Oberkochen, Germany) microscope. In each section, we measured the distance from the pial surface to white matter. Three measures were taken from each section, and at least six sections were analyzed per animal. These values were averaged to obtain cortical thickness in individual animals.
Density of neurons. NeuN-stained sections were examined with a confocal microscope (Leica, Nussloch, Germany) using a $40 \times$ oil immersion objective. Neurons were counted in three-dimensional counting boxes $(250 \times 250 \times 12 \mu \mathrm{m})$ positioned in either layer II/III or layer V/VI of primary visual cortex. An average of 1600 neurons were counted for each experimental animal. Neuronal density was calculated by averaging values obtained from at least six sections per animal.

Quantification of GAD65 staining. All confocal images were collected with an Olympus Optical (Tokyo, Japan) confocal microscope using a $60 \times$ oil immersion lens. An initial analysis on stained sections from the different groups was performed to establish settings for laser intensity, gain, offset, and pinhole size. Care was taken to avoid saturation at either end of the pixel intensity range $(0-255)$. Confocal settings were then held constant through the study. Examination of animals from the various treatment groups was interdigitated to avoid bias caused by slow shifts in laser power. Five to eight sections per animal were analyzed. For each section, we imaged three adjacent fields $(105 \times 105 \mu \mathrm{m})$ located in layer II/III of primary visual cortex. In each field, a stack of 10 GAD65 optical sections separated by $1 \mu \mathrm{m}$ was collected at the top face of the tissue section. The image within each stack with the highest average pixel intensity was selected for the quantitative analysis of GAD65 immunoreactivity (Silver and Stryker, 2000; Tropea et al., 2003).

Perisomatic GAD65 signals ("puncta ring") from four to five target neurons were outlined for each image, and GAD65 signal intensity was calculated (MetaMorph; Molecular Devices, Sunnyvale, CA). For each neuron, signal intensity values were divided by the background labeling in the cell soma (which is devoid of GAD65 staining). A total sample of 90-180 neurons was analyzed for each animal.

The area occupied by GAD65-positive pixels in the neuropil was calculated for each image by applying a threshold (average background signal in the cell somas multiplied by four) and masking all blood vessels and cells bodies. Calculation of the cross-sectional area of GAD65 staining was used here and in previous studies (Smith et al., 2000; Tropea et al., 2003) to estimate the density of presynaptic boutons.

Density of WFA-positive cells. Labeled cells were counted (Pizzorusso et al., 2002) blind to the treatment on a fluorescence microscope using a $20 \times$ objective $\left(0.1 \mathrm{~mm}^{2}\right.$ fields). Six to 8 sections were analyzed for each experimental animal.

\section{Immunoblotting}

Rats received unilateral BoNT/E injections at P14, and visual cortices were dissected $1,7,14$, and $21 \mathrm{~d}$ after treatment. Proteins were extracted (Viegi et al., 2002) with lysis buffer (1\% Triton X-100, 10\% glycerol, 20 mм Tris-HCl, pH 7.5, 150 mm NaCl, 10 mм EDTA, 0.1 mм Na $V_{3} O_{4}, 1$ $\mu \mathrm{g} / \mathrm{ml}$ leupeptin, $1 \mu \mathrm{g} / \mathrm{ml}$ aprotinin, and $1 \mathrm{~mm}$ PMSF). Protein extracts $(10 \mu \mathrm{g})$ were separated by electrophoresis and blotted, and filters were incubated with an antibody specific for the BoNT/E-cleaved form of SNAP-25 (1:50 dilution) or with an antibody recognizing both BoNT/Ecleaved and intact SNAP-25 (1:1000; Sternberger Monoclonals, Lutherville, $\mathrm{MD}$ ). Blots were then reacted with HRP-conjugated secondaries (Bio-Rad, Hercules, CA) and developed by ECL (Amersham Biosciences, Little Chalfont, UK). Filters were also probed with anti- $\beta$-tubulin monoclonal antibody (1:500 dilution; Sigma), which serves as an internal standard for protein quantification.

\section{Electrophysiology}

Rats were anesthetized with urethane $(7 \mathrm{ml} / \mathrm{kg} ; 20 \%$ solution in saline, i.p.; Sigma) and placed in a stereotaxic apparatus. Both eyes were fixed by means of adjustable metal rings surrounding the external portion of the eye bulb, and optic disk locations were projected onto a tangent screen to determine the vertical meridian. Body temperature during the experiments was constantly monitored with a rectal probe and maintained at $37^{\circ} \mathrm{C}$ with a heating blanket. Electrocardiogram was also continuously monitored. A portion of the skull overlying the binocular visual cortex was carefully drilled and the dura was removed. A glass micropipette (2 $\mathrm{M} \Omega$ ) filled with $\mathrm{NaCl}(3 \mathrm{M})$ was inserted into the binocular portion of primary visual cortex.

Single-unit recordings. The position of receptive fields of single units were mapped using a hand-held stimulator. Only cells with receptive 
fields within $20^{\circ}$ of the vertical meridian were included in the analysis. Most ( $>80 \%$ ) of the cells in our sample were recorded from supragranular layers (i.e., at a depth $<800 \mu \mathrm{m}$ from the cortical surface). Spontaneous activity and peak response were determined from peristimulus time histograms recorded in response to computer-generated bars, averaged over 10-20 stimulus presentations as described previously (Lodovichi et al., 2000).

Ocular dominance (OD) was evaluated according to the methods of Hubel and Wiesel (1962). Neurons in ocular dominance class 1 were driven only by stimulation of the contralateral eye; neurons in ocular dominance class $2 / 3$ were binocular and preferentially driven by the contralateral eye; neurons in ocular dominance class 4 were equally driven by the two eyes; neurons in ocular dominance class $5 / 6$ were binocular and preferentially driven by the ipsilateral eye; neurons in ocular dominance class 7 were driven only by the ipsilateral eye. For each animal, the bias of the OD distribution toward the contralateral eye [contralateral bias index $(\mathrm{CBI})]$ was calculated as follows: $\mathrm{CBI}=\left[\left(N_{(1)}\right.\right.$ $\left.\left.-N_{(7)}\right)+1 / 2\left(N_{(2 / 3)}-N_{(5 / 6)}\right)+\mathrm{N}_{\text {TOT }}\right] / 2 N_{\text {TOT }}$, where $N_{(i)}$ is the number of cells in class $i$, and $N_{\text {TOT }}$ is the total number of recorded cells in a specific animal.

Visual evoked potential recordings. The electrode was positioned at a depth of $400-500 \mu \mathrm{m}$ into the cortex, in which visual evoked potentials (VEPs) had their maximal amplitude. Transient VEPs were recorded in response to abrupt reversal of a horizontal square-wave grating, generated by computer on a display (Sony, Tokyo, Japan) by a visual stimulus generator card (Cambridge Research System, Rochester, Kent, UK), which was positioned in front of the rat's eyes to include the binocular visual field. Signals were amplified (10,000-fold), bandpass filtered (0.1$500 \mathrm{~Hz}$ ), and fed to a computer for storage and analysis. At least 50 events were averaged in synchrony with the stimulus contrast reversal. Transient VEPs were evaluated in the time domain by measuring the peak-totrough amplitude and the latency of the major negative component (Pizzorusso et al., 2002). VEPs in response to a blank stimulus were also frequently recorded to estimate noise. Visual acuity was measured as the highest spatial frequency that still evoked a response above noise level at maximum contrast.

Analysis of all electrophysiological data were performed by an investigator (L.G.) that was unaware of the experimental treatment of the animals.

\section{Muscimol experiments}

Ten animals at the peak of the critical period (age range, P23-P28; saline, $n=5$; muscimol, $n=5$ ) were used. Muscimol (30 mm; Sigma) was dissolved in saline and stored at $4^{\circ} \mathrm{C}$. In preliminary experiments, we found that muscimol injections into the binocular visual cortex $(1 \mu \mathrm{l})$ were highly effective in blocking activity within $30 \mathrm{~min}$. In all of the animals, a craniotomy was made on both sides of the skull. A sample of 10-12 neurons were recorded from a single pipette track in the right hemisphere. The binocular portion of left visual cortex was then injected with saline or muscimol. After a delay of $30 \mathrm{~min}$, we started to record from single units again during withdrawal along the same track. The experimenter (L.R.) did not know whether muscimol or saline was injected, and the code was broken only at the completion of the analysis.

\section{Behavioral assessment of visual acuity}

Behavioral assessment of visual acuity was performed starting from P60 in animals that received BoNT/E $(n=10)$ or vehicle $(n=7)$ at P14 and in naive subjects $(n=6)$. Rats were tested blind to treatment in the visual water task as described previously (Prusky et al., 2000; Pizzorusso et al., 2006). Briefly, the device consisted of a trapezoidal-shaped pool, filled with water, partially divided at one end in two arms by a divider. Visual stimuli consisted of gratings of various spatial frequencies or gray fields, which were generated with two computer monitors and placed at the end of each arm. A hidden platform was placed below the grating. Animals were released from the nondivided end of the box and trained to associate the stimulus grating with the submerged platform (i.e., escape from water). The position of the grating and the platform was alternated in a pseudorandom sequence. Visual water task trains animals to distinguish initially a low (0.1 cycles/degree) spatial frequency vertical grating from

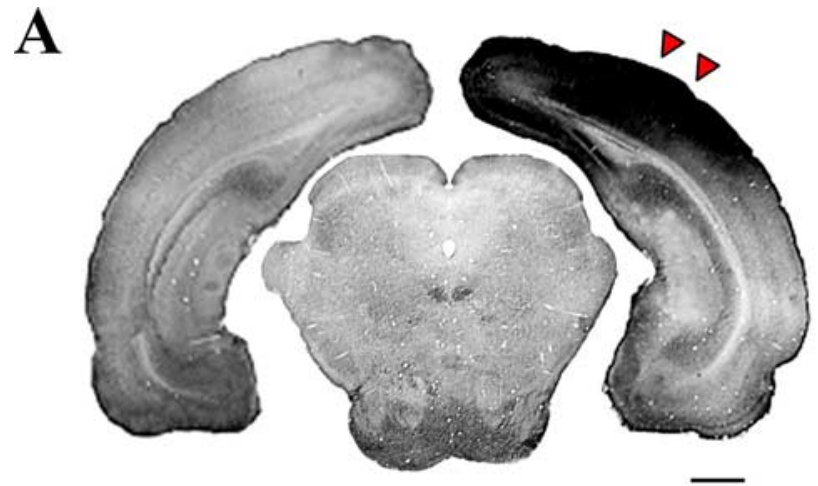

B
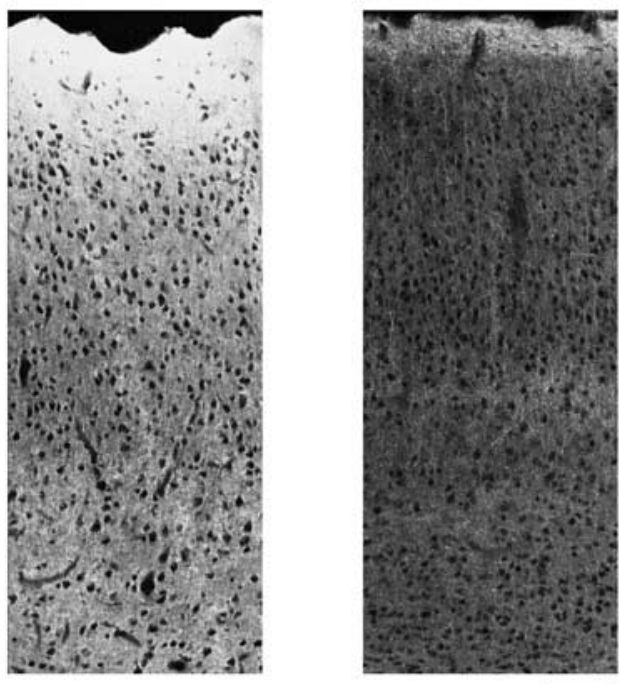

C

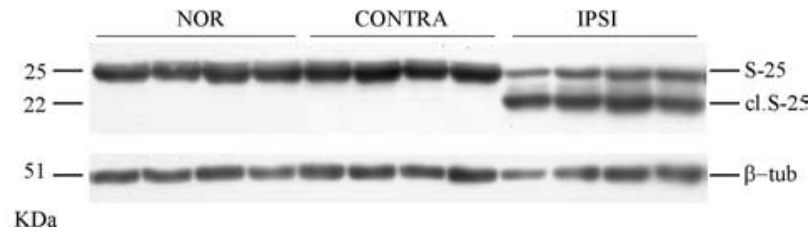

Figure 1. Characterization of BoNT/E effects in the visual cortex. A, Immunostaining for cleaved SNAP-25 (dark labeling) in a coronal section through the occipital cortex and midbrain of a P16 rat, $2 \mathrm{~d}$ after unilateral BoNT/E injection. Cleaved SNAP-25 is evident in the binocular visual cortex (delimited by arrows) of the infused side (right), whereas no signal is detectable in the contralateral hemisphere (left). The superior colliculus is also devoid of staining. Scale bar, 1 $\mathrm{mm}$. B, Confocal images of immunostaining for intact SNAP-25 in the cortex of a P16 rat that received a unilateral BoNT/E infusion $2 \mathrm{~d}$ earlier. Although the uninjected hemisphere (left) displays the normal punctate staining characteristic of SNAP-25, immunoreactivity is virtually completely abolished on the treated side (right). Scale bar, $100 \mu \mathrm{m}$. C, Representative immunoblotting for intact (S-25) and cleaved (cl. S-25) SNAP-25 on cortical protein extracts from P15 rats. Tissues were harvested from normal animals (NOR) and from the side contralateral (CONTRA) and ipsilateral (IPSI) to BoNT/E, $1 \mathrm{~d}$ after infusion. Each lane represents one animal. $\beta$-tub, $\beta$-Tubulin (internal standard).

gray and then tests the limit of this ability at higher spatial frequencies. A trial was recorded as incorrect if an animal entered the arm without the platform. Animals were removed from the pool when they found the platform. The limit of discrimination was estimated by increasing the spatial frequency of the grating until performance fell below $70 \%$ accuracy. The highest spatial frequency at which $70 \%$ accuracy was achieved was recorded as the visual acuity (Prusky et al., 2000; Pizzorusso et al., 2006). 
Statistical analysis

Statistical analysis was performed with SigmaStat (version 3.1; Systat Software, Port Richmond, CA). Differences between two groups were assessed with a two-tailed $t$ test. Differences between three or more groups were evaluated with one-way ANOVA, followed by a Tukey's or Holm-Sidak test for data normally distributed and with Kruskal-Wallis one-way ANOVA with Dunn's post hoc test for data non-normally distributed. Normality of distributions was assessed with KolmogorovSmirnov test. Differences between OD distributions were assessed using a $\chi^{2}$ test (four degrees of freedom). Level of significance was $p<0.05$.

\section{Results}

Transient silencing of cortical activity by BoNT/E

We unilaterally injected BoNT/E (30 nM solution) or vehicle ( $2 \%$ rat serum albumin in PBS) into the visual cortex of rat pups at the time of eye opening (P14). Immunostaining for cleaved SNAP25 , performed $1-4 \mathrm{~d}$ after BoNT/E injection, demonstrated that BoNT/E effects were restricted to the injected side and that diffusion of the toxin was adequate to cover the whole extent of primary visual cortex in the infused hemisphere (Fig. $1 A$ ). Staining for intact SNAP-25 revealed a strong loss of immunoreactivity in the injected area and the normal, punctuate labeling typical of synaptic markers in the contralateral hemisphere (Fig. $1 B$ ). There was no detectable spread of BoNT/E effects to more ventral structures such as the superior colliculus (Fig. $1 A$ ) and hippocampus (data not shown). To further confirm the regional specificity of BoNT/E effects, we performed immunoblot analysis on protein extracts from the treated and untreated visual cortex at P15 (i.e., $1 \mathrm{~d}$ after injection), using an antibody that recognizes both intact and BoNT/E-truncated SNAP-25. The results were clear in indicating that SNAP-25 cleavage only occurred on the infused side (Fig. 1C) and that levels of intact SNAP-25 in the contralateral hemisphere were comparable with those of normal animals (post ANOVA Tukey's test, $p>0.05$ ).

Extracellular recordings of spiking activity and VEPs were used to demonstrate functional consequences of SNAP-25 cleavage. Recordings at P16-P17 showed that infusion of vehicle had no effect on visual responses (data not shown). Conversely, BoNT/E produced a blockade of neuronal activity in the injected hemisphere (Fig. $2 A, B$ ). No visually responsive cells and no measurable VEP responses could be found in the cortical area surrounding the injection sites (Fig. $2 B$ ). The silencing effects of BoNT/E were consistently limited to the injected side, and indeed vigorous responses could be recorded in the contralateral hemisphere (Fig. $2 A, B$ ).

Duration of BoNT/E action was assessed by recordings from visual cortex at different times after delivery of the toxin. At P21P23 (i.e., 7-9 d after BoNT/E) cortical cell discharges were still blocked in the infused hemisphere, and no VEP responses could be elicited (Fig. 3A). Visually responsive cells began to reappear at P28 in four of five recorded animals. Accordingly, VEP recordings showed sluggish field potential responses (with significantly increased latency, $p<0.05 t$ test vs normal animals) (Fig. $3 A$ ) in these rats and ongoing blockade in the remaining animal. Visual activity was completely recovered by P35, i.e., $21 \mathrm{~d}$ after BoNT/E injection (Fig. 3A). Primary visual cortex was dissected from the recorded animals to analyze the time course of SNAP-25 cleavage. In keeping with the electrophysiological results, Western blotting demonstrated very high levels of cleaved SNAP-25 at P15 and P21, i.e., 1-7 d after BoNT/E (Fig. 3B-D). A decrease in BoNT/E-truncated SNAP-25 and a corresponding rise in intact SNAP-25 was observed in P28 animals (Fig. 3B-D). Cleaved SNAP-25 was no longer detectable at P35 (Fig. 3B-D). Thus, BoNT/E effects are completely extinguished $21 \mathrm{~d}$ after injection.
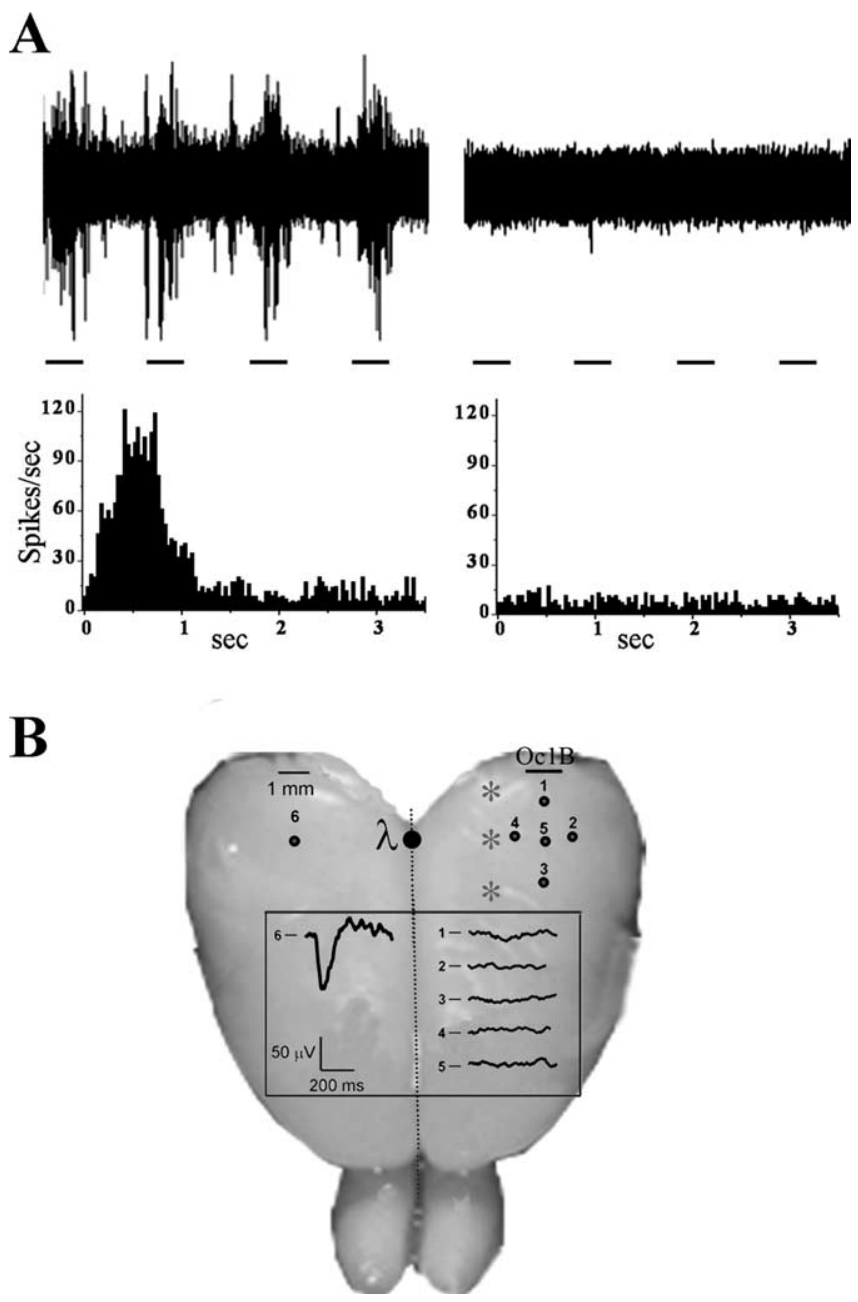

Figure 2. Functional consequences of BoNT/E infusion. $\boldsymbol{A}$, Top, Representative extracellular recordings of spiking activity from the binocular cortex ipsilateral (right) and contralateral (left) to BoNT/E infusion, $2 \mathrm{~d}$ after toxin injection. Passage of a light-bar stimulus across the visual field is indicated by the horizontal bars under the trace (each horizontal bar, $1.90 \mathrm{~s}$ ). Bottom, Examples of peristimulus time histograms recorded in the infused (right) and contralateral (left) cortex. Visual response is abolished in the BoNT/E-treated hemisphere. B, Representative physiological mapping of the effects of BoNT/E in a P17 rat (i.e., $3 \mathrm{~d}$ after injection). The BoNT/E injection sites (asterisks) and the location of six microelectrode penetrations are shown on a dorsal view of the rat brain. Five penetrations (numbered 1-5) were made in the binocular visual cortex $(0 \mathrm{C} 1 \mathrm{~B})$ of the infused hemisphere and showed no VEP response (see traces in the box). A robust field potential response (track number six) could be evoked from the uninjected side. $\lambda$, Lambda suture.

To exclude that the long-term blockade of activity might have deleterious effects on neuronal survival in the cortex, we determined cortical thickness and neuron density at P35. This analysis revealed no changes in the BoNT/E-injected and contralateral cortex with respect to either the normal or vehicle-injected P35 cortex (one-way ANOVA, $p>0.19$ for all comparisons) (Fig. $4 A-C$ ).

Silencing of one side results in bilateral impairments in visual cortical development

Functional development of binocular striate cortex was assessed at P34-P36, i.e., around the end of the critical period for rat visual cortex (Fagiolini et al., 1994). Data were collected from four groups: (1) naive cortex, (2) cortex infused with vehicle, and cortex (3) ipsilateral and (4) contralateral to BoNT/E injection.

We first measured the maturation of spatial resolution (acu- 
ity) in the cortex using VEPs (Fagiolini et al., 1994; Huang et al., 1999; Gianfranceschi et al., 2003). We found that visual acuity was approximately half of the normal value on both the injected and uninjected side of P35 animals treated with BoNT/E (one-way ANOVA, $p<0.001$; post hoc Tukey's test, $p<0.05$ ) (Fig. 5), whereas vehicle injections had no effect $(p>0.05)$. Acuity values were indistinguishable in the two hemispheres of BoNT/E-infused rats (post ANOVA Tukey's test, $p>0.05$ ). Thus, a unilateral silencing of striate cortex results in bilateral impairments in the development of visual acuity.

Single-unit recordings were performed to evaluate cortical binocularity in BoNT/ E-treated and control rats. This analysis indicated that the OD of cortical neurons was not affected by the activity blockade. OD histograms and CBIs were normal in both the BoNT/E-injected and contralateral hemisphere ( $\chi^{2}$ test, $p>0.05$; oneway ANOVA, $p>0.3$ ) (Fig. $6 A, B$ ).

Visual cortex maturation in rats is accompanied by a decline in the potential for experience-dependent plasticity (Fagiolini et al., 1994; Pizzorusso et al., 2002). We assessed sensitivity to MD in a group of BoNT/E-infused and control rats. We found that 4-5 d of MD starting from P34-P36 were ineffective in inducing a change in eye preference in normal and vehicle-infused rats (Fig. $7 A, B$ ). OD histograms and CBIs of these animals were superimposable to those measured in undeprived rats $\left(p>0.05 ; \chi^{2}\right.$ test and twotailed $t$ test, respectively). In contrast, a robust OD shift to the open eye could be observed in both the ipsilateral and contralateral hemisphere of BoNT/E-injected rats (Fig. $7 A, B$ ). For this analysis, BoNT/E-infused animals were split into two groups and monocularly deprived either contralateral or ipsilateral to the injection, to assess plasticity of the treated and untreated hemisphere, respectively. Statistical testing demonstrated that CBI values were lower in the BoNT/E-injected and contralateral cortex compared with normal and vehicle-infused controls (one-way ANOVA, $p<0.001$; post hoc Tukey's test, $p<0.05$ ). CBIs in the former groups did not differ (post ANOVA Tukey's test, $p>$ 0.05 ), indicating that the magnitude of the OD shift was similar in the two hemispheres of BoNT/E rats. We conclude that unilateral blockade of cortical activity prolongs the critical period for plasticity in both hemispheres.

Substantial evidence indicates that intracortical GABAmediated inhibition is an important determinant of the critical period (Hensch et al., 1998; Huang et al., 1999; Hensch, 2005). We analyzed the expression of GAD65, an isoform of glutamic acid decarboxylase that is concentrated in presynaptic terminals, in layers II-III of striate cortex by quantitative confocal microscopy (Huang et al., 1999). Measurement of the area occupied by the immunoreactive staining indicated that both the cortex ipsilateral and contralateral to BoNT/E injection had a reduced density of GAD65 terminals in the neuropil (one-way ANOVA, $p<$ 0.001 ; post hoc Holm-Sidak test, $p<0.05$ with respect to naive

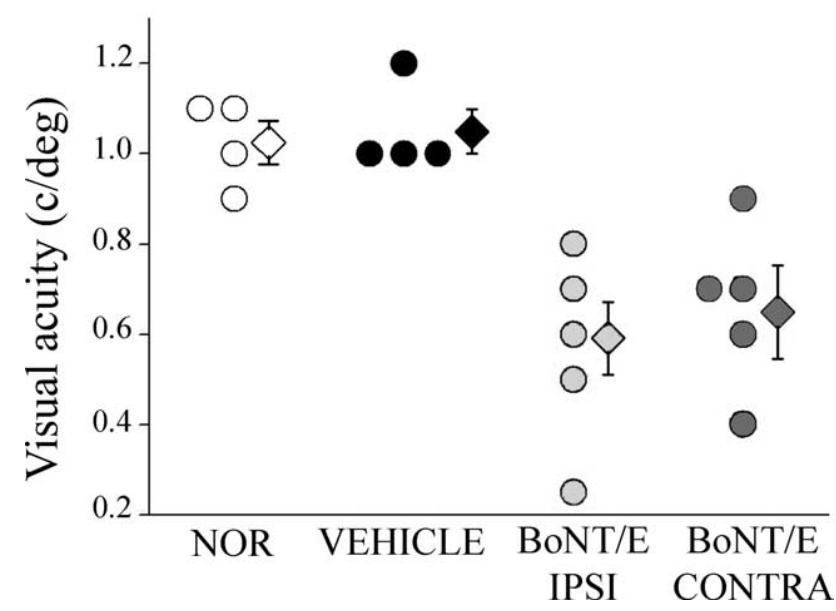

Figure 5. Bilateral impairments in spatial resolution in P35 BoNT/E rats. Summary of visual acuities in all animal groups. Each circle represents one animal. Mean visual acuity (diamonds) is significantly reduced in both hemispheres of BoNT/E rats compared with that in normal or vehicle-injected animals. Error bars indicate SE. Abbreviations as in Figure 3.

and vehicle-infused cortex) (Fig. 8A). Analysis of GAD65 fluorescence in puncta rings surrounding the soma of cortical neurons also indicated a lower GAD65 signal in both hemispheres of the BoNT/E-treated rats (one-way ANOVA on ranks, $p<0.001$; post hoc Dunn's test, $p<0.05$ ) (Fig. $8 B$ ). 

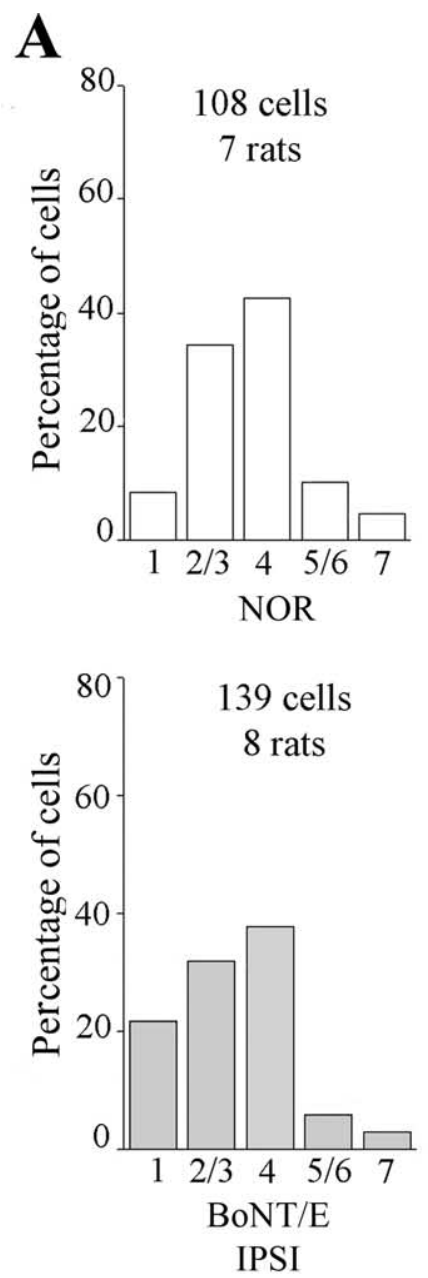

B

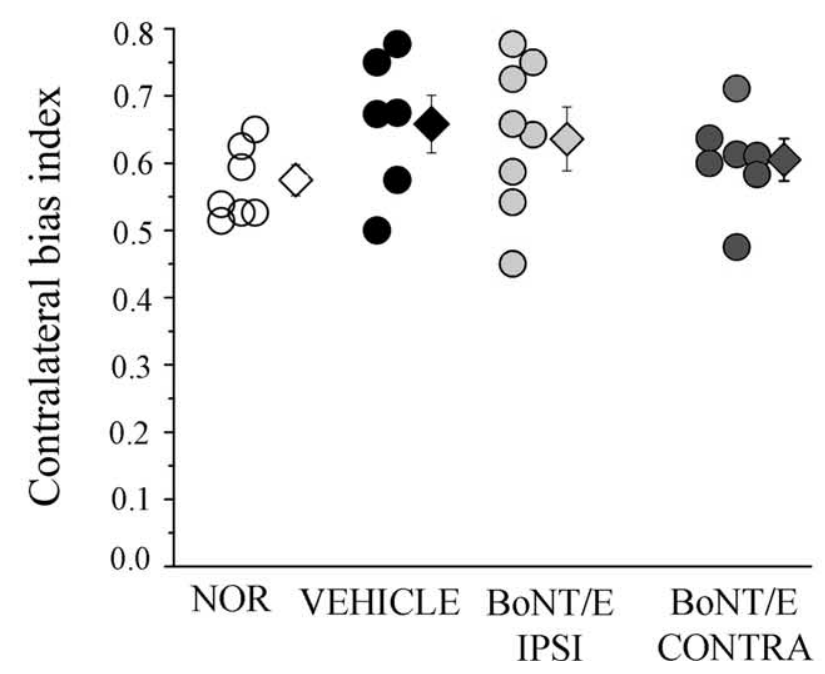

Figure 6. Activity blockade has no effects on cortical binocularity. A, OD distributions of control and BoNT/E rats at P35. Control rats were either naive (NOR) or injected with vehicle at P14 (VEHICLE). BoNT/E rats were injected at P14 and recorded both ipsilateral and contralateral to the infusion side (BoNT/E IPSI and BoNT/E CONTRA). Number of animals and cells as indicated. $\boldsymbol{B}$, CBI of control and BoNT/E-treated rats at P35. Circles, Values of individual animals; diamonds, mean of the group. Error bars indicate SE and, when not seen, are within the symbol. No differences in $\mathrm{OD}$ can be detected among the groups (one-way ANOVA, $p>0.3$ ).
A
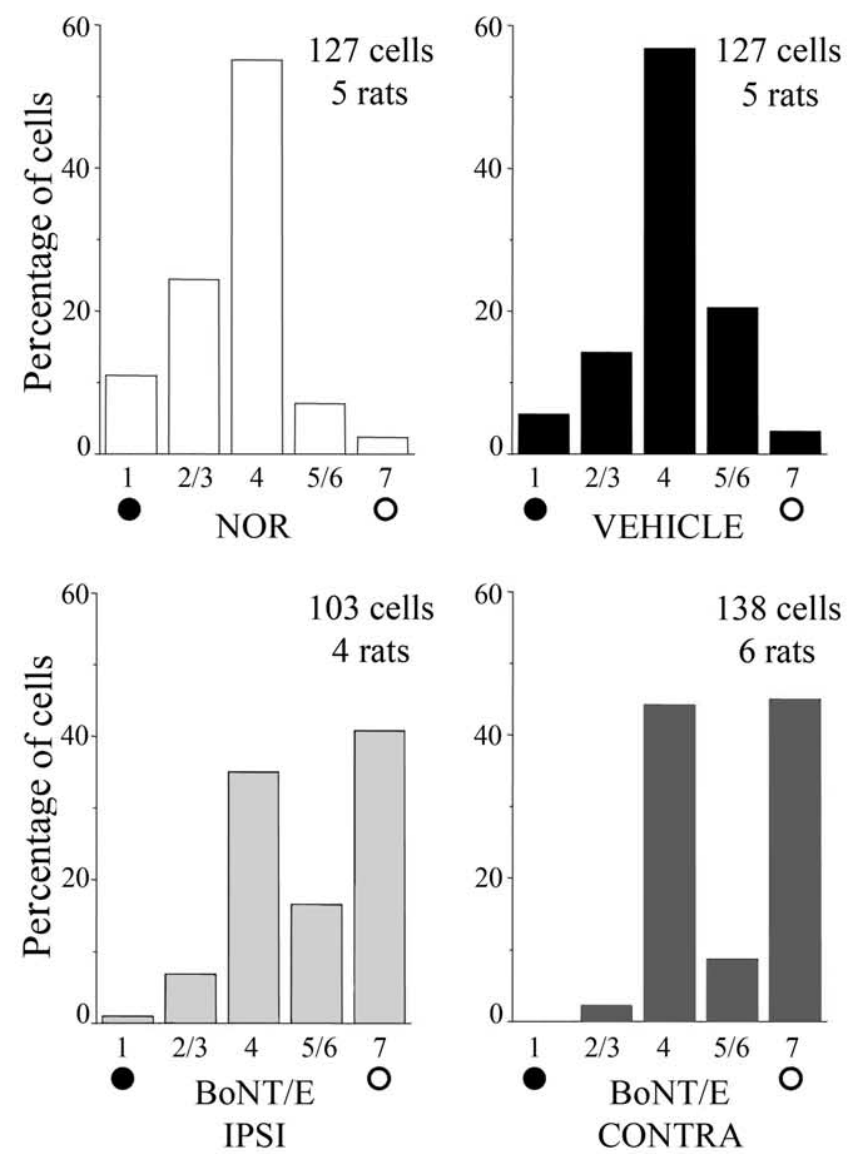

B

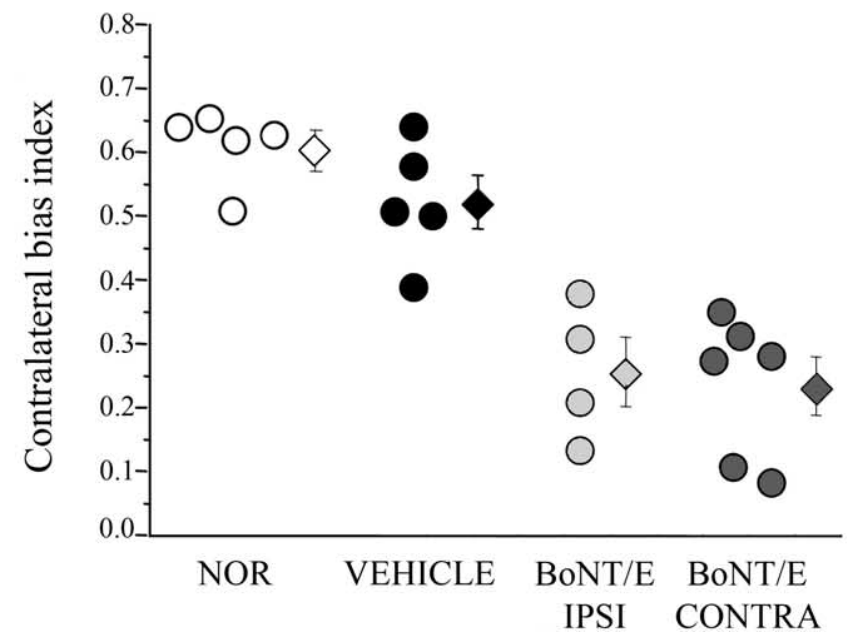

Figure 7. Bilateral prolongation of the critical period in $\mathrm{P} 35$ BoNT/E rats. $A, O D$ distributions in normal and vehicle- and BoNT/E-injected rats subjected to $M D$ at $P 35.0 D$ was assessed after $4-5 \mathrm{~d}$ of monocular occlusion. The deprived eye was always the eye contralateral to the cortical hemisphere being examined. Both hemispheres of BoNT/E rats display a significant shift toward the nondeprived eye (open circles). Number of animals and cells as indicated. Note that OD histograms for BoNT/E animals look bimodal, suggesting that two subsets of cortical cells are differentially sensitive to the activity blockade. $\boldsymbol{B}$, CBI for all animals recorded in each experimental group (circles, values of single animals; diamonds, mean $\pm \mathrm{SE}$ ). MD effects are consistently detectable in both hemispheres of BoNT/E-injected rats. Abbreviations as in Figure 6. 
A

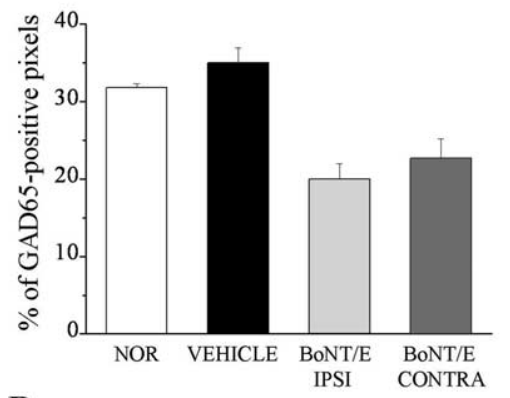

B

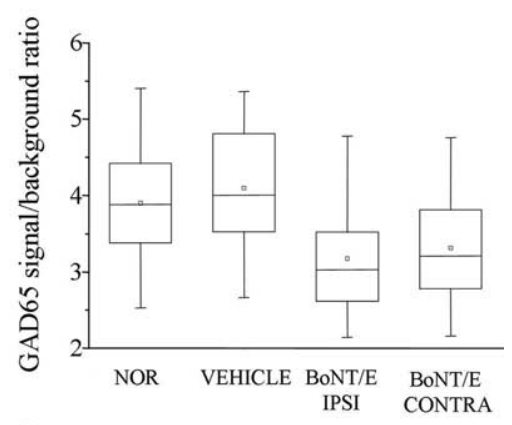

C

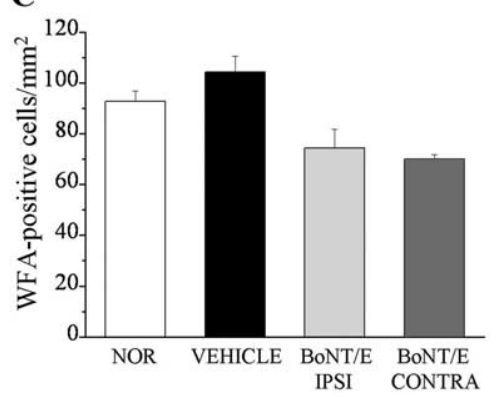

Figure 8. Bilateral reductions in markers of intracortical inhibition in P35 BoNT/E rats. $\boldsymbol{A}$, Mean density of GAD65-positive pixels in the neuropil (layers II-III) is significantly reduced in the cortex ipsilateral and contralateral to BoNT/E infusion with respect to both naive and vehicle-injected cortex. Error bars indicate SE. B, Quantification of GAD65 immunofluorescence in GABAergic terminals around the soma of target neurons. The box chart summarizes the distribution of the GAD65 signal-to-background ratio (intensity of GAD65 label in puncta rings divided by the background staining in the soma) for the cells in each experimental group. The horizontal lines in the box denote the 25th, 50th, and 75th percentile values. The error bars denote the 5 th and 95 th percentile values. The square symbol in the box denotes the mean of the column of data. GAD65 staining is less intense in both hemispheres of BoNT/E-injected rats compared with normal and vehicle-injected cortex (one-way ANOVA followed by Dunn's test, $p<0.05)$. C, Mean \pm SE density of perineuronal nets surrounded neurons in layer II/III. Density of WFA-positive cells is reduced ipsilateral and contralateral to BoNT/E infusion. Abbreviations as in Figure 6.

Cessation of OD plasticity in rats correlates with the developmental organization into perineuronal nets of chondroitin sulfate proteoglycans (Pizzorusso et al., 2002). We assessed the number of perineuronal nets by staining cortical sections with WFA. Countings of WFA-positive cells in layers II-III at P35 revealed a decrease in the BoNT/E-injected hemisphere with respect to both the naive and vehicle-infused cortex (one-way ANOVA, $p<0.01$; post hoc Holm-Sidak test, $p<0.05$ ) (Fig. $8 C$ ). The number of perineuronal nets was similarly reduced in the cortex contralateral to BoNT/E infusion (post ANOVA HolmSidak test, $p<0.05)$ (Fig. 8C).
A

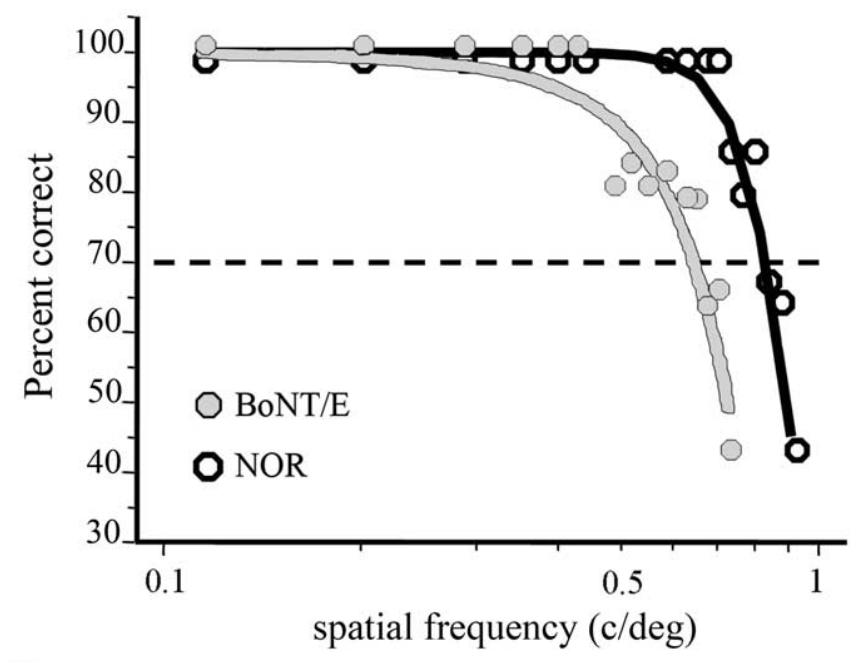

B

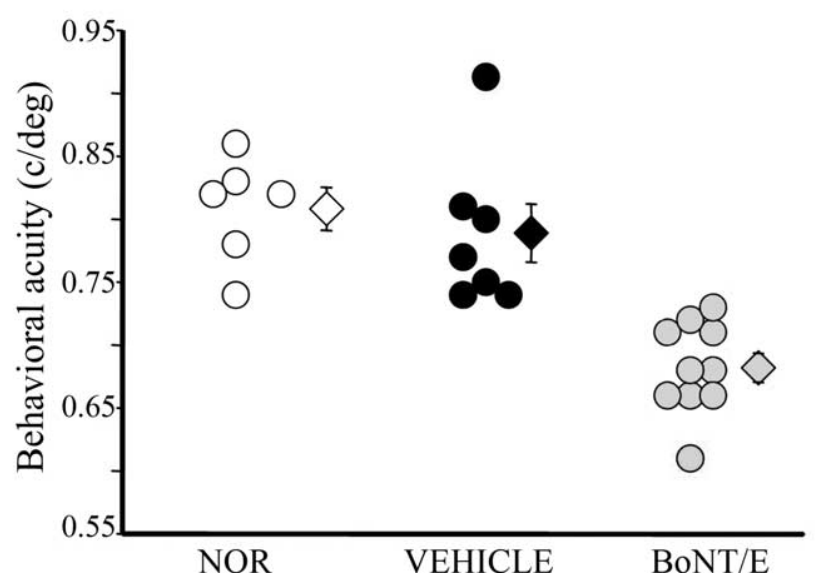

Figure 9. Deficits in behavioral acuity in adult BoNT/E rats. $A$, Representative frequency-ofseeing curves for a normal rat (white symbols) and a BoNT/E rat (gray symbols). Each point is the average performance at a spatial frequency. A sigmoid curve is fit to the data, and the point at which the curve intersects $70 \%$ accuracy is taken as the grating threshold. B, Summary of data on behavioral visual acuity. Each circle represents one animal. Mean \pm SE visual acuity (diamonds) is significantly reduced in BoNT/E animals with respect to normal or vehicle-injected rats (ANOVA; post hoc Holm-Sidak test, $p<0.01$ ).

\section{Transient blockade of activity leaves a lasting change in} visual cortex

The impaired spatial resolution and the sensitivity to MD found in P35 BoNT/E rats might either represent a delay in developmental maturation of the visual cortex or persist into adulthood. To address this issue, a group of control rats and rats injected with BoNT/E at P14 were allowed to survive until P60-P100 (i.e., $30-70 \mathrm{~d}$ after the completion of BoNT/E effects) and tested behaviorally for the determination of visual acuity. Using a twoalternative forced-choice discrimination task (visual water box) (Prusky et al., 2000), we found significantly lower visual acuities in the animals treated with BoNT/E with respect to normal and vehicle-injected rats (post ANOVA Holm-Sidak test, $p<0.01$ ) (Fig. 9A,B). Thus, BoNT/E rats exhibit a persistent deficit in visual acuity at the behavioral level. To determine whether spatial resolution was equally impaired in both hemispheres of adult $\mathrm{BoNT} / \mathrm{E}$ rats, the animals were recorded for the determination of 


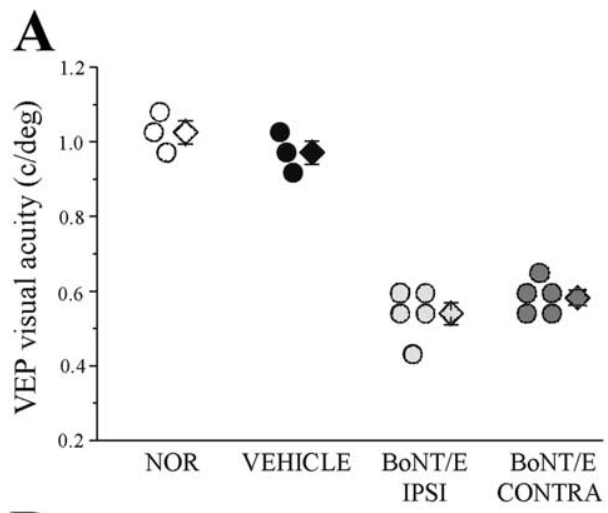

B

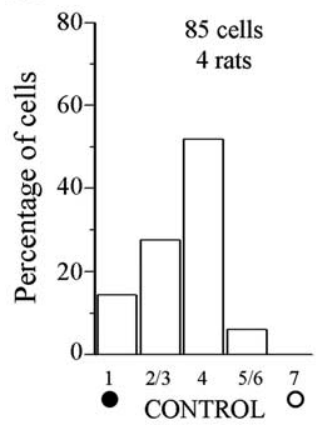

C

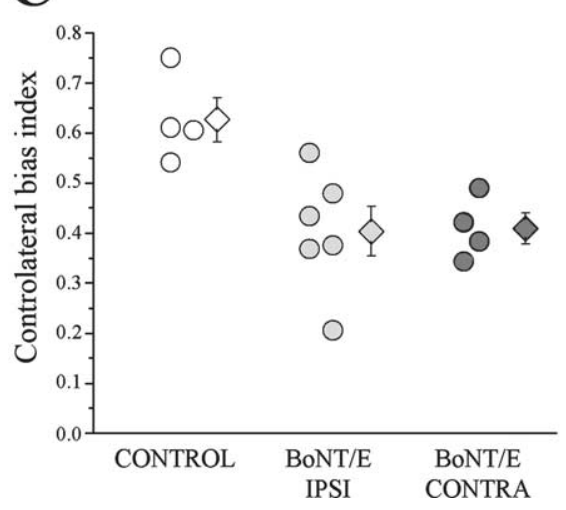

Figure 10. Persistent bilateral impairments in visual acuity and enduring plasticity in adult BoNT/E rats. A, VEP visual acuity of P60 -P100 animals. Acuity is poor in both hemispheres of BoNT/E-treated rats (circles, single animal values; diamonds, mean). Error bars indicate SE and, when not seen, are within the symbol. $B, 0 D$ distributions in animal older than P60 subjected to MD. The control group includes one vehicle-injected rat and three normal animals. In each hemisphere, $O D$ was assessed after $4-5 \mathrm{~d}$ of occlusion of the contralateral eye. An OD shift in favor of the open eye (open circles) is observed in both hemispheres of BoNT/E-treated rats but not in the control group. Number of animals and cells as indicated. C, CBI for all animals recorded in each experimental group (circles, values of single animals; diamonds, mean $\pm \mathrm{SE}$ ). CBI values are lower than normal in both hemispheres of BoNT/E-injected rats (post ANOVA HolmSidak test, $p<0.05$ ). Abbreviations as in Figure 6.

VEP acuity. The results were clear in indicating that visual acuity was low both ipsilateral and contralateral to BoNT/E injection (one-way ANOVA, $p<0.001$; post hoc Tukey's test, $p<0.01$ vs normal and vehicle rats) (Fig. 10A). Acuity values did not differ between the two hemispheres of BoNT/E rats $(p>0.05)$. We conclude that a unilateral blockade of cortical activity during the sensitive period produces bilateral impairments in visual function that extend into adulthood.

Plasticity was assessed with 4-5 d of MD in a second group of P60-P80 rats. Monocular occlusion had no effect in control rats but significantly skewed the OD distribution toward the open eye

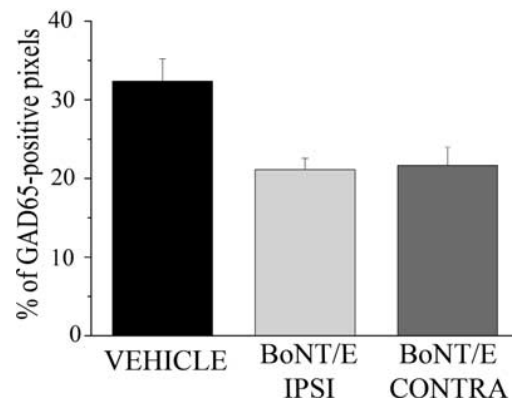

Figure 11. Persistent bilateral downregulation of inhibition in adult BoNT/E rats. Mean density of GAD65-positive pixels in the neuropil (layers II-III) of rats older than P100. The density of presynaptic inhibitory terminals is significantly reduced in the cortex ipsilateral (IPSI) and contralateral (CONTRA) to BoNT/E infusion with respect to the vehicle-injected cortex. Error bars indicate $S E$.

in both the BoNT/E-injected and contralateral cortex $\left(\chi^{2}\right.$ test, $p<0.05$ ) (Fig. $10 B$ ). Analysis of CBI values of individual rats indicated that the OD shift was comparable in the two hemispheres of BoNT/E rats (one-way ANOVA, $p=0.01$; post hoc Holm-Sidak test, $p>0.05$ ) (Fig. 10C). Thus, the two hemispheres retained a similar potential for plasticity in adult age.

To assess whether a persistent downregulation of intracortical inhibition might explain this enduring plasticity, coronal cortical sections from vehicle- and BoNT/E-injected rats (older than P100) were reacted with anti-GAD65 antibodies. A quantitative confocal analysis revealed that both hemispheres of BoNT/Etreated rats had a reduced density of presynaptic inhibitory boutons in the neuropil with respect to vehicle-injected rats (layers II-III; one-way ANOVA, $p=0.011$; post hoc Holm-Sidak test, $p<0.05$ ) (Fig. 11). Analysis of GAD65 fluorescence in puncta rings surrounding the cell body of target neurons also revealed similar bilateral reductions of GAD65 staining in BoNT/E rats (data not shown).

\section{Reduction of spontaneous activity levels after silencing of the} contralateral hemisphere

The dramatic long-term consequences of abolishing transcallosal input point to a crucial role for interhemispheric interactions in shaping cortical development. To begin addressing the mechanisms responsible for these effects, we performed single-unit recordings in the untreated hemisphere of BoNT/E-treated rats, $7 \mathrm{~d}$ after toxin infusion (i.e., during the period of blockade). The analysis revealed that, although responses to light-bar stimuli remained extremely brisk (Fig. $3 A$ ), there was a remarkable decrease in the spontaneous discharge of cortical cells. Spontaneous firing rate had a median value of 3.37 spikes/s (interquartile ranges, 2.16-6.57) in the untreated hemisphere of BoNT/E rats. These values were substantially lower than those found in normal age-matched animals (median, 13.03 spikes/s; interquartile ranges, 7.03-18.92). The difference was highly significant (Man$\mathrm{n}$-Whitney rank sum test, $p<0.001$ ). To determine whether this reduction in spontaneous activity reflects a developmental plastic event or it is rather an acute effect of callosal deprivation, we performed an experiment in which single cortical units were recorded in the same animal before and after silencing of the contralateral hemisphere. BoNT/E was not suited for this kind of experiment, because the toxin requires several hours to become fully active (our unpublished experiments). Therefore, we selected the $\mathrm{GABA}_{\mathrm{A}}$ agonist muscimol as the blocking agent. Approximately $10-12$ cortical cells were recorded along a micropipette penetration and muscimol $(30 \mathrm{~mm})$ or saline as control was 
injected into contralateral striate cortex. We found that the muscimol, but not saline, blocked cortical activity within $30 \mathrm{~min}$. After this period, we started to record from single units again during withdrawal along the same track. We found a clear reduction of spontaneous activity levels after muscimol, but not saline, infusion (Fig. 12A). Quantification of spontaneous discharge indicated that cells recorded after muscimol had a significantly reduced firing frequency (one-way ANOVA on ranks, $p<0.001$; post Dunn's test, $p<0.05$ vs all of the other groups) (Fig. $12 \mathrm{~B}$ ). The other experimental conditions did not differ from each other (post ANOVA Dunn's test, $p>0.05$ ) (Fig. 12B). These data demonstrate reductions of spontaneous discharge after blockade of the contralateral hemisphere.

\section{Discussion}

In this study, we exploited the clostridial neurotoxin BoNT/E to investigate the effects of a unilateral, transient blockade of cortical activity during the sensitive period. BoNT/E is a highly selective metalloprotease that elicits persistent but reversible inhibition of transmitter release via cleavage of the synaptic protein SNAP-25 (Schiavo et al., 2000; Costantin et al., 2005; Davletov et al., 2005). Proteolysis of SNAP-25 by BoNT/E prevents the formation of a functional SNARE (soluble NSF-attachment protein receptor) complex, resulting in a highly selective blockade of synaptic activity (Schiavo et al., 2000; Montecucco and Molgo, 2005). The action of BoNT/E is completely reversible, because the protease is inactivated with time and intact SNAP-25 is wholly replenished (Keller et al., 1999; Costantin et al., 2005). Thus, BoNT/E combines a potent and long-lasting action with full reversibility at the cellular level (Bozzi et al., 2006). These features make BoNT/E an ideal tool to address the role of synaptic activity in the brain. Indeed, a single administration of the toxin is sufficient to switch off activity for a period of 2 weeks, thus avoiding the need for more invasive techniques such as long-term minipump infusion of activity blockers (tetrodotoxin and muscimol), which is often impractical in small developing rodents. Importantly, we found no adverse effects of BoNT/E on neuronal survival, consistent with previous reports (Osen-Sand et al., 1996).

Differently from tetanus toxin, BoNT/E is not axonally transported (Lalli et al., 2003). We made several controls to make sure that there was no unintended diffusion or transport of the toxin from the injection site. These controls rely on the detection of intact and cleaved SNAP-25 by immunohistochemistry and immunoblotting (Fig. 1). Following the fate of BoNT/E in vivo is inherently difficult because of the very tiny amounts of injected toxin. In this context, detection of cleaved substrate molecules provides the most sensitive assay for tracking movement of BoNT/E. Indeed, single toxin molecules can cleave a large number of SNAP-25 target molecules, providing a dramatic amplifying effect.

We have shown that intracortical delivery of BoNT/E at the time of eye opening (P14) results in strictly unilateral silencing of activity until near the peak of the critical period for visual cortex, i.e., P25-P28 in rats (Fagiolini et al., 1994; Lodovichi et al., 2000). Considerable evidence indicates that eye opening represents a crucial event for refinement of cortical circuitry (Maffei et al., 2004; Gandhi et al., 2005). Thus, injections of BoNT/E at P14 allow to target the process of cortical maturation since its initial phases.

There are three main findings from this study: (1) a transient unilateral silencing of intrinsic cortical activity produces deficits in visual acuity and an extension of the critical period for OD
A
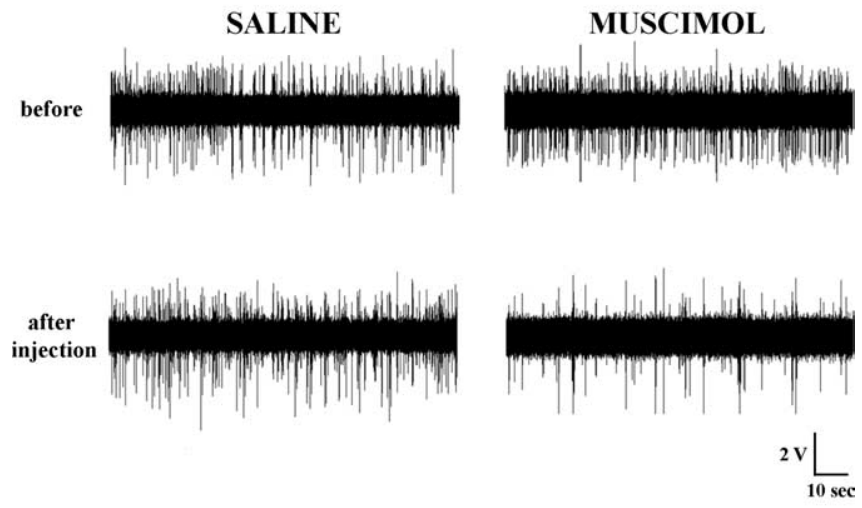

\section{B}

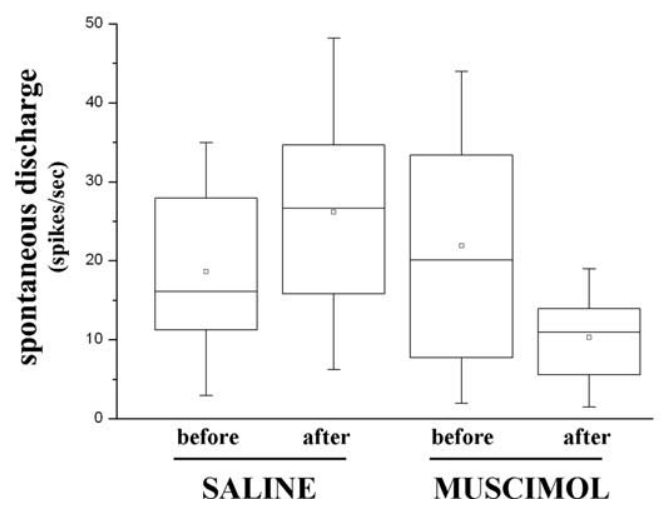

Figure 12. Reduced neuronal firing after blockade of the opposite hemisphere. $\boldsymbol{A}$, Representative recordings of spontaneous spiking activity from visual cortical neurons, before and after muscimol (or saline) injection into the contralateral hemisphere. $\boldsymbol{B}$, Quantification of spontaneous firing rates before and after muscimol (or saline) infusion into the contralateral hemisphere. The box chart summarizes the distribution of spontaneous activity for the cells in each experimental group (saline, $n=51$ cells; muscimol, $n=61$ cells). The horizontal lines in the box denote the 25 th, 50 th, and 75 th percentile values. The error bars denote the 5 th and 95 th percentile values. The square symbol in the box denotes the mean of the column of data. Spontaneous discharge is significantly reduced after muscimol delivery to the opposite side (one-way ANOVA, $p<0.001$, followed by Dunn's test, $p<0.05$ ).

plasticity in the blocked hemisphere; (2) these same effects are detectable in the striate cortex of the opposite side, pointing to a crucial role for interhemispheric connections in postnatal development; and (3) impairments in cortical maturation persist into adulthood, indicating that a transient blockade of neural discharges leaves a permanent trace in cortical circuitry.

Our data demonstrate the requirement for intrinsic activity in several aspects of visual cortex development. It was shown previously that silencing of action potentials completely prevents maturation of orientation selectivity and clustering of horizontal connections in ferret visual cortex (Chapman and Stryker, 1993; Ruthazer and Stryker, 1996). We show here that a transient blockade of cortical activity causes a reduction of visual acuity and an extension of the period of susceptibility to MD. These effects are accompanied by a decrease in GAD65 immunoreactivity. A reduced inhibitory tone may explain the deficits in spatial resolution and prolongation of the plastic state. Indeed, substantial evidence indicates that intracortical inhibitory connections control maturation of visual function and critical period plasticity (Huang et al., 1999; Berardi et al., 2003; Hensch, 2005). For 
example, a reduction of GABA-mediated transmission in visual cortex accounts for some of the classic effects of dark rearing (Gianfranceschi et al., 2003; Iwai et al., 2003). Changes at the level of excitatory circuitry, such as alterations in AMPA or NMDA receptors (He et al., 2006), could also underlie the effects of the activity blockade. This possibility remains to be evaluated. Interestingly, we found no effects of BoNT/E on the OD of cortical neurons. It is possible that BoNT/E affects OD but that the return of intrinsic activity is sufficient to restore normal binocularity by the time the recordings were performed (P35).

A second major conclusion from this study is that interhemispheric communication plays a fundamental role in functional development of the cortex. The callosal pathway matures quite early in development and links retinotopically corresponding loci in the two hemispheres (Lewis and Olavarria, 1995; Olavarria, 1996). Interhemispheric linkages serve important functions, including binding together the separate representations of the two halves of the visual field (Berlucchi and Rizzolatti, 1968; Engel et al., 1991). We have shown here a significant reduction in cortical activity after silencing of the contralateral side. This is consistent with previous studies in cats indicating that callosal projections provide a kind of general sustaining input to the contralateral hemisphere (Blakemore et al., 1983; Yinon et al., 1992). This activity deprivation might explain the impaired cortical development on the side contralateral to BoNT/E injection. Indeed, the striate cortex contralateral to the blockade exhibits poor spatial resolution, prolongation of the critical period for OD plasticity, and reductions in markers of intracortical inhibition. It is worth noting that the magnitude of these effects is comparable in the blocked and untreated hemisphere. Thus, maturation of the blocked cortex is superimposable to that of the opposite side, which only lacks callosal input and maintains normal afferent activity through the direct retinogeniculate pathway. The very similar developmental deficits observed ipsilateral and contralateral to the activity blockade indicate a fundamental role for callosal linkages in coordinating the process of cortical maturation. In teleological terms, parallel development of the two sides of the brain is needed to ensure a match in information processing between the cerebral hemispheres. Indeed, this coordinated maturation grants uniform sampling of visual information and the ability to combine the two partial cortical maps into a single, coherent representation.

In rats, the whole extent of primary visual cortex contains numerous callosal cells and terminals (Olavarria and Van Sluyters, 1985; Lewis and Olavarria, 1995). This organization is different from that observed in higher mammals such as cats and primates, in which callosal terminals are mainly concentrated at the border between area 17 and 18 (Olavarria, 1996; Houzel and Milleret, 1999). This raises the issue of whether callosal influences are as important for cortical development in higher mammals as reported here for the rat. Reports in the literature suggest that this may indeed be the case. Early section of the callosum in cats produces a reduction in behaviorally measured visual acuity, supporting a role for interhemispheric communication in cortical maturation (Elberger, 1984). Monkeys that received unilateral lesions of primary visual cortex in infancy display impairments of stimulus detection in the intact visual hemifield (Moore et al., 1996, their Fig. 3). Development of other functional properties might rely more on retinothalamic input and/or local intracortical plasticity rather than on callosal influences. For example, development of orientation selectivity in ferret visual cortex is not affected by activity blockade in the contralateral hemisphere (Chapman and Stryker, 1993).
A third important finding of our study is that a transient activity blockade leaves a lasting change in visual cortex. Indeed, we observed basically no recovery of visual acuity in either hemisphere of animals injected with BoNT/E at P14 and allowed to survive until P60-P100. In these adult animals, both the previously blocked and the uninjected hemisphere maintained a similar susceptibility to monocular suture. The data indicate that silencing neural activity produces persistent effects on visual function and the cortical plasticity machinery. It has been shown recently that adult plasticity is shaped by previous experience (Hofer et al., 2006). Our data demonstrate that malleability of adult circuits can be conditioned by activity deprivation during early life. The persistent sensitivity to MD found on the site opposite to the blockade indicates that lack of callosal input during development is sufficient to maintain the adult cortex in a plastic state.

Several recent findings in mouse visual cortex suggest that at least some measure of OD plasticity persists into adulthood (Sawtell et al., 2003; Tagawa et al., 2005; Hofer et al., 2006), making one wonder whether the susceptibility to MD reported here for the adult BoNT/E rats reflects at least some of this normal, extended plasticity. We consider this interpretation unlikely, however, because we and others have consistently failed to reveal MD effects in normal adult rats by both single-unit and VEP recordings (Fagiolini et al., 1994; Guire et al., 1999; present results).

In summary, blocking intrinsic cortical activity produces developmental changes that are more profound and long-lasting than those induced by manipulations of afferent sensory input. We also revealed a previously unknown functional matching in the developmental maturation of the two cerebral hemispheres. Such coordinated development allows homogeneous processing of sensory information coming from the two visual hemifields, thus ensuring consistency of the visual percept. The importance of the callosal pathway in integrating cerebral processing is still apparent in adults. Patients with unilateral occipital cortex injury show reduced spatial and temporal sensitivities in the sighted hemifield (Hess and Pointer, 1989; Rizzo and Robin, 1996).

\section{References}

Benevento LA, Bakkum BW, Port JD, Cohen RS (1992) The effects of darkrearing on the electrophysiology of the rat visual cortex. Brain Res 572:198-207.

Berardi N, Pizzorusso T, Maffei L (2000) Critical periods during sensory development. Curr Opin Neurobiol 10:138-145.

Berardi N, Pizzorusso T, Ratto GM, Maffei L (2003) Molecular basis of plasticity in the visual cortex. Trends Neurosci 26:369-378.

Berlucchi G, Rizzolatti G (1968) Binocularly driven neurons in visual cortex of split-chiasm cats. Science 159:308-310.

Blakemore C, Diao YC, Pu ML, Wang YK, Xiao YM (1983) Possible functions of the interhemispheric connexions between visual cortical areas in the cat. J Physiol (Lond) 337:331-349.

Bozzi Y, Costantin L, Antonucci F, Caleo M (2006) Action of botulinum neurotoxins in the central nervous system: antiepileptic effects. Neurotox Res 9:197-203.

Chapman B, Stryker MP (1993) Development of orientation selectivity in ferret visual cortex and effects of deprivation. J Neurosci 13:5251-5262.

Costantin L, Bozzi Y, Richichi C, Viegi A, Antonucci F, Funicello M, Gobbi M, Mennini T, Rossetto O, Montecucco C, Maffei L, Vezzani A, Caleo M (2005) Antiepileptic effects of botulinum neurotoxin E. J Neurosci 25:1943-1951.

Davletov B, Bajohrs M, Binz T (2005) Beyond BOTOX: advantages and limitations of individual botulinum neurotoxins. Trends Neurosci 28:446-452.

Desai NS, Cudmore RH, Nelson SB, Turrigiano GG (2002) Critical periods for experience-dependent synaptic scaling in visual cortex. Nat Neurosci 5:783-789.

Elberger AJ (1984) The existence of a separate, brief critical period for the 
corpus callosum to affect visual development. Behav Brain Res 11:223-231.

Engel AK, Konig P, Kreiter AK, Singer W (1991) Interhemispheric synchronization of oscillatory neuronal responses in cat visual cortex. Science 252:1177-1179.

Fagiolini M, Pizzorusso T, Berardi N, Domenici L, Maffei L (1994) Functional postnatal development of the rat primary visual cortex and the role of visual experience: dark rearing and monocular deprivation. Vision Res 34:709-720.

Fox K, Wong RO (2005) A comparison of experience-dependent plasticity in the visual and somatosensory systems. Neuron 48:465-477.

Gandhi SP, Cang J, Stryker MP (2005) An eye-opening experience. Nat Neurosci 8:9-10.

Gianfranceschi L, Siciliano R, Walls J, Morales B, Kirkwood A, Huang ZJ, Tonegawa S, Maffei L (2003) Visual cortex is rescued from the effects of dark rearing by overexpression of BDNF. Proc Natl Acad Sci USA 100:12486-12491.

Guire ES, Lickey ME, Gordon B (1999) Critical period for the monocular deprivation effect in rats: assessment with sweep visually evoked potentials. J Neurophysiol 81:121-128.

He HY, Hodos W, Quinlan EM (2006) Visual deprivation reactivates rapid ocular dominance plasticity in adult visual cortex. J Neurosci 26:2951-2955.

Hensch TK (2005) Critical period plasticity in local cortical circuits. Nat Rev Neurosci 6:877-888.

Hensch TK, Fagiolini M, Mataga N, Stryker MP, Baekkeskov S, Kash SF (1998) Local GABA circuit control of experience-dependent plasticity in developing visual cortex. Science 282:1504-1508.

Hess RF, Pointer JS (1989) Spatial and temporal contrast sensitivity in hemianopia. A comparative study of the sighted and blind hemifields. Brain 112:871-894.

Hofer SB, Mrsic-Flogel TD, Bonhoeffer T, Hubener M (2006) Prior experience enhances plasticity in adult visual cortex. Nat Neurosci 9:127-132.

Houzel JC, Milleret C (1999) Visual inter-hemispheric processing: constraints and potentialities set by axonal morphology. J Physiol (Paris) 93:271-284.

Huang ZJ, Kirkwood A, Pizzorusso T, Porciatti V, Morales B, Bear MF, Maffei L, Tonegawa S (1999) BDNF regulates the maturation of inhibition and the critical period of plasticity in mouse visual cortex. Cell 98:739-755.

Hubel DH, Wiesel TN (1962) Receptive fields, binocular interaction and functional architecture in the cat's visual cortex. J Physiol (Lond) 160:106-154.

Iwai Y, Fagiolini M, Obata K, Hensch TK (2003) Rapid critical period induction by tonic inhibition in visual cortex. J Neurosci 23:6695-6702.

Katz LC, Shatz CJ (1996) Synaptic activity and the construction of cortical circuits. Science 274:1133-1138.

Keller JE, Neale EA, Oyler G, Adler M (1999) Persistence of botulinum neurotoxin action in cultured spinal cord cells. FEBS Lett 456:137-142.

Kirkwood A, Rioult MC, Bear MF (1996) Experience-dependent modification of synaptic plasticity in visual cortex. Nature 381:526-528.

Lalli G, Bohnert S, Deinhardt K, Verastegui C, Schiavo G (2003) The journey of tetanus and botulinum neurotoxins in neurons. Trends Microbiol 11:431-437.

Lewis JW, Olavarria JF (1995) Two rules for callosal connectivity in striate cortex of the rat. J Comp Neurol 361:119-137.

Li Y, Fitzpatrick D, White LE (2006) The development of direction selectivity in ferret visual cortex requires early visual experience. Nat Neurosci 9:676-681.

Lodovichi C, Berardi N, Pizzorusso T, Maffei L (2000) Effects of neurotrophins on cortical plasticity: same or different? J Neurosci 20:2155-2165.

Maffei A, Nelson SB, Turrigiano GG (2004) Selective reconfiguration of layer 4 visual cortical circuitry by visual deprivation. Nat Neurosci 7:1353-1359.
Montecucco C, Molgo J (2005) Botulinal neurotoxins: revival of an old killer. Curr Opin Pharmacol 5:274-279.

Moore T, Rodman HR, Repp AB, Gross CG, Mezrich RS (1996) Greater residual vision in monkeys after striate cortex damage in infancy. J Neurophysiol 76:3928-3933.

Morales B, Choi SY, Kirkwood A (2002) Dark rearing alters the development of GABAergic transmission in visual cortex. J Neurosci 22:8084-8090.

Olavarria J, Van Sluyters RC (1985) Organization and postnatal development of callosal connections in the visual cortex of the rat. J Comp Neurol 239:1-26.

Olavarria JF (1996) Non-mirror-symmetric patterns of callosal linkages in areas 17 and 18 in cat visual cortex. J Comp Neurol 366:643-655.

Osen-Sand A, Staple JK, Naldi E, Schiavo G, Rossetto O, Petitpierre S, Malgaroli A, Montecucco C, Catsicas S (1996) Common and distinct fusion proteins in axonal growth and transmitter release. J Comp Neurol 367:222-234.

Pizzorusso T, Medini P, Berardi N, Chierzi S, Fawcett JW, Maffei L (2002) Reactivation of ocular dominance plasticity in the adult visual cortex. Science 298:1248-1251.

Pizzorusso T, Medini P, Landi S, Baldini S, Berardi N, Maffei L (2006) Structural and functional recovery from early monocular deprivation in adult rats. Proc Natl Acad Sci USA 103:8517-8522.

Prusky GT, West PW, Douglas RM (2000) Behavioral assessment of visual acuity in mice and rats. Vision Res 40:2201-2209.

Rizzo M, Robin DA (1996) Bilateral effects of unilateral visual cortex lesions in human. Brain 119:951-963.

Ruthazer ES, Stryker MP (1996) The role of activity in the development of long-range horizontal connections in area 17 of the ferret. J Neurosci 16:7253-7269.

Sawtell NB, Frenkel MY, Philpot BD, Nakazawa K, Tonegawa S, Bear MF (2003) NMDA receptor-dependent ocular dominance plasticity in adult visual cortex. Neuron 38:977-985.

Schiavo G, Montecucco C (1995) Tetanus and botulism neurotoxins: isolation and assay. Methods Enzymol 248:643-652.

Schiavo G, Matteoli M, Montecucco C (2000) Neurotoxins affecting neuroexocytosis. Physiol Rev 80:717-766.

Sherman SM, Spear PD (1982) Organization of visual pathways in normal and visually deprived cats. Physiol Rev 62:738-855.

Silver MA, Stryker MP (2000) Distributions of synaptic vesicle proteins and GAD65 in deprived and nondeprived ocular dominance columns in layer IV of kitten primary visual cortex are unaffected by monocular deprivation. J Comp Neurol 422:652-664.

Smith TD, Adams MM, Gallagher M, Morrison JH, Rapp PR (2000) Circuit-specific alterations in hippocampal synaptophysin immunoreactivity predict spatial learning impairment in aged rats. J Neurosci 20:6587-6593.

Sur M, Leamey CA (2001) Development and plasticity of cortical areas and networks. Nat Rev Neurosci 2:251-262.

Tagawa Y, Kanold PO, Majdan M, Shatz CJ (2005) Multiple periods of functional ocular dominance plasticity in mouse visual cortex. Nat Neurosci 8:380-388.

Tropea D, Caleo M, Maffei L (2003) Synergistic effects of brain-derived neurotrophic factor and chondroitinase $\mathrm{ABC}$ on retinal fiber sprouting after denervation of the superior colliculus in adult rats. J Neurosci 23:7034-7044.

Viegi A, Cotrufo T, Berardi N, Mascia L, Maffei L (2002) Effects of dark rearing on phosphorylation of neurotrophin Trk receptors. Eur J Neurosci 16:1925-1930.

Yinon U, Chen M, Gelerstein S (1992) Binocularity and excitability loss in visual cortex cells of corpus callosum transected kittens and cats. Brain Res Bull 29:541-552. 